PROCEEDINGS OF THE

AMERICAN MATHEMATICAL SOCIETY

Volume 139, Number 8, August 2011, Pages 2995-3008

S 0002-9939(2011)10626-9

Article electronically published on March 23, 2011

\title{
ON ZERO-DIMENSIONALITY AND THE CONNECTED COMPONENT OF LOCALLY PSEUDOCOMPACT GROUPS
}

\author{
D. DIKRANJAN AND GÁBOR LUKÁCS
}

(Communicated by Alexander N. Dranishnikov)

Dedicated to Wis Comfort on the occasion of his 78th birthday

\begin{abstract}
A topological group is locally pseudocompact if it contains a nonempty open set with pseudocompact closure. In this paper, we prove that if $G$ is a group with the property that every closed subgroup of $G$ is locally pseudocompact, then $G_{0}$ is dense in the component of the completion of $G$, and $G / G_{0}$ is zero-dimensional. We also provide examples of hereditarily disconnected pseudocompact groups with strong minimality properties of arbitrarily large dimension, and thus show that $G / G_{0}$ may fail to be zero-dimensional even for totally minimal pseudocompact groups.
\end{abstract}

\section{INTRODUCTION}

A Tychonoff space is zero-dimensional if it has a base consisting of clopen (openand-closed) sets. With each topological group $G$ there are associated functorial subgroups related to the connectedness properties of $G$, defined as follows (cf. [11, 1.1.1]):

(a) $G_{0}$ denotes the connected component of the identity;

(b) $q(G)$ denotes the quasi-component of the identity, that is, the intersection of all clopen sets containing the identity;

(c) $z(G)$ denotes the intersection of all kernels of continuous homomorphisms from $G$ into zero-dimensional groups;

(d) $o(G)$ denotes the intersection of all open subgroups of $G$.

It is well known that these subgroups are closed and normal (cf. [19, 7.1], [12, $2.2]$, and [22, 1.32(b)]). Clearly, $G_{0} \subseteq q(G) \subseteq z(G) \subseteq o(G)$, and all four are equal for locally compact groups.

Theorem 1.1 ([19, 7.7, 7.8]). Let $L$ be a locally compact group. Then $L / L_{0}$ is zero-dimensional and $L_{0}=q(L)=z(L)=o(L)$.

Received by the editors February 15, 2010 and, in revised form, May 30, 2010.

2010 Mathematics Subject Classification. Primary 22A05, 54D25, 54H11; Secondary 22D05, 54D05, 54D30.

The first author acknowledges the financial aid received from SRA, grants P1-0292-0101, J1-9643-0101, and MTM2009-14409-C02-01.

The second author gratefully acknowledges the generous financial support received from NSERC and the University of Manitoba, which enabled him to do this research.

(C)2011 American Mathematical Society

Reverts to public domain 28 years from publication 
The aim of the present paper is to investigate to what extent the condition of local compactness can be relaxed in Theorem 1.1. Although Theorem 1.1 might appear as a result about connectedness, it has far more to do with different degrees of disconnectedness. Following [18, we say that a space $X$ is hereditarily disconnected if its connected components are singletons, and $X$ is totally disconnected if its quasicomponents are singletons. Clearly,

zero-dimensional $\stackrel{(*)}{\Longrightarrow}$ totally disconnected $\stackrel{(* *)}{\Longrightarrow}$ hereditarily disconnected,

and by Vedenissoff's classic theorem, both implications are reversible for locally compact (Hausdorff) spaces; that is, the three properties are equivalent for such spaces (cf. [36]).

It is well known that the quotient $G / G_{0}$ is hereditarily disconnected for every topological group $G$ (cf. [19, 7.3] and [22, 1.32(c)]). Thus, if the implications $(*)$ and $(* *)$ are reversible for $G / G_{0}$, then $G / G_{0}$ is zero-dimensional, and so $G_{0}=q(G)=z(G)$. If in addition $z(G)=o(G)$, then Theorem 1.1 holds for $G$. This phenomenon warrants introducing some terminology.

Definition 1.2. A topological group $G$ is Vedenissoff if the quotient $G / G_{0}$ is zerodimensional; if in addition $z(G)=o(G)$, then we say that $G$ is strongly Vedenissoff.

Our goal is to identify classes of (strongly) Vedenissoff groups and to find examples of non-Vedenissoff groups that have many compactness-like properties. The latter will demonstrate how close a group must be to being locally compact (or compact) in order to be Vedenissoff. (Not every Vedenissoff group is strongly Vedenissoff. Indeed, $\mathbb{Q} / \mathbb{Z}$ is zero-dimensional, but has no proper open subgroups, and so $z(\mathbb{Q} / \mathbb{Z}) \neq o(\mathbb{Q} / \mathbb{Z})$. However, thanks to Theorem 2.7 (a) below, these two notions coincide in the class of groups that are considered in this paper.)

A Tychonoff space $X$ is pseudocompact if every continuous real-valued map on $X$ is bounded. A topological group $G$ is locally pseudocompact if there is a neighborhood $U$ of the identity such that $\operatorname{cl}_{G} U$ is pseudocompact. (Clearly, every metrizable locally pseudocompact group is locally compact.) We say that $G$ is hereditarily [locally] pseudocompact if every closed subgroup of $G$ is [locally] pseudocompact. (Note that the adjective hereditarily applies only to closed subgroups here, and not to all subgroups. Indeed, by Corollary 2.6 below, if every subgroup of a topological group is locally pseudocompact, then the group is discrete, which is of no interest for the present paper.) More than fifteen years ago, Dikranjan proved the following theorem.

Theorem 1.3 ([10, 1.2, 2.6]). Let $G$ be a hereditarily pseudocompact group. Then $G / G_{0}$ is zero-dimensional, $G_{0}=q(G)$, and $G_{0}$ is dense in $(\widetilde{G})_{0}$; that is, $G$ is strongly Vedenissoff.

We obtain in this paper a theorem that simultaneously generalizes Theorems 1.1 and 1.3 and provides a positive solution to a problem posed by Comfort and Lukács (cf. [3, 4.13]).

Theorem A. Let $G$ be a hereditarily locally pseudocompact group. Then $G / G_{0}$ is zero-dimensional and $G_{0}=q(G)=z(G)=o(G)$; that is, $G$ is strongly Vedenissoff.

The next example shows that the condition of hereditary local pseudocompactness in Theorem $\mathrm{A}$ cannot be replaced with (local) pseudocompactness. 
Example 1.4. Comfort and van Mill showed that for every natural number $n$ there exists an abelian pseudocompact group $G_{n}$ such that $G_{n}$ is totally disconnected, but $\operatorname{dim} G_{n}=n$ (cf. [5, 7.7]). In particular, the converse of the implication (*) may fail for these groups $G_{n}$, and they are not Vedenissoff. This shows that pseudocompact groups need not be Vedenissoff.

Although pseudocompactness alone is too weak a property to imply that the group is Vedenissoff, it turns out that it is sufficient in the presence of some additional compactness-like properties. Recall that a (Hausdorff) topological group $G$ is minimal if there is no coarser (Hausdorff) group topology (cf. 31] and [16]), and $G$ is totally minimal if every (Hausdorff) quotient of $G$ is minimal (cf. [13]). Equivalently, $G$ is totally minimal if every continuous surjective homomorphism $G \rightarrow H$ is open.

An unpublished result of Shakhmatov states that the converse of $(*)$ holds for minimal pseudocompact groups. Specifically, Shakhmatov proved that every pseudocompact totally disconnected group admits a coarser zero-dimensional group topology, and thus minimal pseudocompact totally disconnected groups are zerodimensional (cf. [10, 1.6]). We prove a generalization of Shakhmatov's result:

\section{Theorem B.}

(a) Every locally pseudocompact totally disconnected group admits a coarser zero-dimensional group topology.

(b) Every minimal, locally pseudocompact, totally disconnected group is zerodimensional and thus strongly Vedenissoff.

Theorem C. Let $G$ be a totally minimal locally pseudocompact group. Then $G_{0}=q(G)$ if and only if $G / G_{0}$ is zero-dimensional, in which case $G$ is strongly Vedenissoff.

Local pseudocompactness cannot be omitted from Theorem B Indeed, more than twenty years ago, Arhangel'skiu asked whether every totally disconnected topological group admits a coarser zero-dimensional group topology. Megrelishvili answered this question in the negative by constructing a minimal totally disconnected group that is not zero-dimensional (cf. [23]). In particular, the converse of the implication $(*)$ fails for minimal groups.

Our last result is a negative one, and it is a far reaching extension of the result of Comfort and van Mill cited in Example 1.4. Recall that a group $G$ is perfectly (totally) minimal if the product $G \times H$ is (totally) minimal for every (totally) minimal group $H$ (cf. 32]).

Theorem D. For every natural number $n$ or $n=\omega$, there exists an abelian pseudocompact group $G_{n}$ such that $G_{n}$ is perfectly totally minimal, hereditarily disconnected, but $\operatorname{dim} G_{n}=n$.

There are many known examples of pseudocompact groups for which the equality $G_{0}=q(G)$ fails (cf. [9, Theorem 11], [11, 1.4.10], and [3, 4.4(d), 5.6]). By Theorem $\mathbb{C}$, one has $\left(G_{n}\right)_{0} \neq q\left(G_{n}\right)$ for each of the groups $G_{n}$ provided by Theorem D, and thus the $G_{n}$ are not totally disconnected. This shows that the converse of the implication $(* *)$ may fail for totally minimal pseudocompact groups.

The paper is structured as follows. In $₫ 2$, we recall some well-known facts on locally pseudocompact and locally compact groups, their $G_{\delta}$-topologies, and their 
connectedness properties. The proofs of Theorems $\mathrm{B}$ and $\mathrm{C}$ are also presented in 92. We devote $\$ 3$ to the proof of Theorem A Finally, in 4 , we prove a general theorem concerning the embedding of groups with minimality properties as quasicomponents of pseudocompact groups with the same minimality properties, which yields Theorem $\mathrm{D}$.

\section{Preliminaries on LOCALly PSEUdocompaCt GRoups}

All topological groups here are assumed to be Hausdorff, and thus Tychonoff (cf. [19, 8.4] and [22, 1.21]). Except when specifically noted, no algebraic assumptions are imposed on the groups; in particular, our groups are not necessarily abelian. A "neighborhood" of a point means an open set containing the point.

Although, in general, there are a number of useful uniform structures on a topological group that induce its topology, in this note, we adhere to the two-sided uniformity and the notions of precompactness and completeness that derive from it (cf. [30], 37], 28], [19, (4.11)-(4.15)], and [22, Section 1.3]). A fundamental property of this notion of completeness is that for every topological group $G$ there is a complete topological group $\widetilde{G}$ (unique up to a topological isomorphism) that contains $G$ as a dense topological subgroup; in other words, $\widetilde{G}$ is a group completion of $G$ (cf. [28] and [22, 1.46]).

Theorem 2.1 ([22, 1.49(a), 1.51]).

(a) Let $G$ be a topological group, and $H$ a subgroup. Then $\widetilde{H}=\mathrm{cl}_{\widetilde{G}} H$.

(b) If $G$ is a locally compact group, then $G$ is complete, that is, $\widetilde{G}=G$.

A subset $X$ of a topological group $G$ is precompact if for every neighborhood $U$ of the identity, there is a finite $S \subseteq X$ such that $X \subseteq(S U) \cap(U S)$. (Some authors refer to precompact sets as bounded ones.) A topological group $G$ is locally precompact if $G$ admits a base of precompact neighborhoods at the identity. Since every pseudocompact subset of a topological group is precompact (cf. [7, 1.11]), locally pseudocompact groups are locally precompact.

Weil showed in 1937 that the completion of a locally precompact group with respect to its left or right uniformity admits the structure of a locally compact group containing $G$ as a dense topological subgroup (cf. 37]). This (one-sided) Weilcompletion coincides with the Răkov-completion $\widetilde{G}$ constructed in 1946 (cf. 28]). Therefore, $G$ is locally precompact if and only if $\widetilde{G}$ is locally compact.

Theorem 2.2 below, which summarizes the main results of [6] and [7, provides a characterization of (locally) pseudocompact groups. Recall that a $G_{\delta}$-subset of a space $(X, \mathcal{T})$ is a set of the form $\bigcap_{n<\omega} U_{n}$ with each $U_{n} \in \mathcal{T}$. The $G_{\delta}$-topology on $X$ is the topology generated by the $G_{\delta}$-subsets of $(X, \mathcal{T})$. A subset of $X$ is $G_{\delta}$-open (respectively, $G_{\delta}$-closed, $G_{\delta}$-dense) if it is open (respectively, closed, dense) in the $G_{\delta}$-topology on $X$.

Theorem 2.2 ([6] and [7]). A topological group $G$ is [locally] pseudocompact if and only if $G$ is [locally] precompact and $G_{\delta}$-dense in $\widetilde{G}$, in which case $\widetilde{G}=\beta G$ $[\beta \widetilde{G}=\beta G]$. In particular, every precompact locally pseudocompact group is pseudocompact.

Since the $G_{\delta}$-topology of groups plays an important role in the present work, we introduce some notation and then record a few useful facts. We let $\Lambda(G)$ denote the 
set of closed $G_{\delta}$-subgroups of the topological group $G$, that is, closed subgroups of $G$ that are also $G_{\delta}$-subsets of $G$, and we set $\Lambda_{c}(G):=\{K \in \Lambda(G) \mid K$ is compact $\}$ and $\Lambda_{c}^{*}(G):=\left\{K \in \Lambda_{c}(G) \mid K \triangleleft G\right\}$.

Theorem 2.3. Let $G$ be a topological group. Then:

(a) $([22,2.5])$ the $G_{\delta}$-topology is a group topology on $G$;

(b) $([19,8.7])$ if $G$ is locally compact, then $\Lambda_{c}(G)$ is a base at the identity for the $G_{\delta}$-topology on $G$;

(c) $([19,8.7])$ if $G$ is locally compact and $\sigma$-compact, then $\Lambda_{c}^{*}(G)$ is a base at the identity for the $G_{\delta}$-topology on $G$.

We have already mentioned that the adjective hereditarily used in the term hereditarily locally pseudocompact applies only to the closed subgroups of a given group. The next theorem shows that it would be uninteresting to interpret hereditarily as applying to all subgroups.

Theorem 2.4. Let $G$ be a locally pseudocompact group. If every countable subgroup of $G$ is locally pseudocompact, then $G$ is discrete.

Before we proceed to the proof of Theorem 2.4, we formulate a well-known observation that will be frequently used later on too.

Lemma 2.5. Let $H$ be a topological group, $D$ a dense subgroup, and $O$ an open subgroup of $H$. Then $\mathrm{cl}_{H}(D \cap O)=O$.

Proof. Since $D$ is dense and $O$ is open in $H$, one has $\operatorname{cl}_{H} O=\operatorname{cl}_{H}(D \cap O)$. On the other hand, $O$ is closed in $H$, because every open subgroup of a topological group is also closed (cf. [19, 5.5]). This completes the proof.

Proof of Theorem 2.4. The proof consists of two steps.

Step 1. The completion $L:=\widetilde{G}$ is locally compact, and so by Theorem 2.3(b), $\Lambda_{c}(L)$ is a base at the identity of the $G_{\delta}$-topology on $L$. Pick $K \in \Lambda_{c}(L)$. Since $G$ is locally pseudocompact, by Theorem $2.2, G$ is $G_{\delta}$-dense in $L$. By Theorem 2.3(a), the $G_{\delta}$-topology is a group topology on $L$, and thus, by Lemma 2.5] $P:=K \cap G$ is $G_{\delta}$-dense in $K$. Let $S$ be a countable subgroup of $P$. By our assumption, $S$ is locally pseudocompact, and by Theorem 2.2, $S$ is pseudocompact. Thus, $S$ is finite, because there are no countably infinite homogeneous pseudocompact spaces (cf. [17, $1.3]$ ), and so $P$ is finite. In particular, $K$ is finite. Therefore, $L$ has a countable pseudocharacter, and hence $L$ is metrizable (cf. [18, 3.3.4]). Consequently, $G$ is metrizable.

Step 2. Let $S$ be a countable subgroup of $G$. By our assumption, $S$ is locally pseudocompact, and thus it is locally compact, because $G$ is metrizable. Consequently, by the Baire Category Theorem, $S$ is discrete. Therefore, every countable subgroup of the metrizable group $G$ is discrete. Hence, $G$ is discrete, as desired.

Corollary 2.6. If every subgroup of a topological group $G$ is locally pseudocompact, then $G$ is discrete.

Next, we summarize the relationship between connectedness properties of locally pseudocompact groups and their completions.

Theorem 2.7. Let $G$ be a locally pseudocompact group. Then:

(a) $([8,1.4],[3,4.5]) ~ q(G)=o(G)=(\widetilde{G})_{0} \cap G$; 
(b) $(33],[3,4.8]) G$ is zero-dimensional if and only if $\widetilde{G}$ is zero-dimensional;

(c) $([8,1.7],[3,4.11(\mathrm{~b})]) G / G_{0}$ is zero-dimensional if and only if $G_{0}$ is dense in $(\widetilde{G})_{0}$, in which case $G_{0}=q(G)$.

We turn to proving Theorems $\mathrm{B}$ and $\mathrm{C}$, which follow from Theorem 2.7. Recall that a group topology is linear if it admits a base at the identity consisting of subgroups. Since every open subgroup is also closed, every linear group topology is zero-dimensional. We prove a slightly stronger version of Theorem $B$,

\section{Theorem B].}

(a) Every locally pseudocompact totally disconnected group admits a coarser linear group topology.

(b) Every minimal, locally pseudocompact, totally disconnected group has a linear topology, and thus it is strongly Vedenissoff.

Proof. (a) The family of open subgroups in a topological group is closed under conjugation and formation of finite intersections. Since $G$ is totally disconnected, $q(G)=\{e\}$, and since $G$ is locally pseudocompact, by Theorem $2.7(\mathrm{a}), q(G)=o(G)$. Thus, $o(G)=\{e\}$. Therefore, the family of open subgroups in $G$ forms a base at the identity for a Hausdorff group topology on $G$, and it is obviously coarser than the topology of $G$. Clearly, this topology is linear.

(b) follows from (a) and the definition of minimality.

Theorem C. Let $G$ be a totally minimal locally pseudocompact group. Then $G_{0}=q(G)$ if and only if $G / G_{0}$ is zero-dimensional, in which case $G$ is strongly Vedenissoff.

Proof. Suppose that $G_{0}=q(G)$. Then the quotient $G / G_{0}=G / q(G)$ is minimal, locally pseudocompact, and totally disconnected. Thus, by Theorem $\mathrm{B}(\mathrm{b}), G / G_{0}$ is zero-dimensional. By Theorem 2.7 (a), $q(G)=o(G)$, and in particular, $z(G)=o(G)$. Therefore, $G$ is strongly Vedenissoff, as required. The converse follows by Theorem 2.7(c).

In the definition of a linear topology, we asked for a base at the identity consisting of open subgroups. Some authors define the same term by requiring the existence of a base at the identity consisting of open normal subgroups. This stronger property warrants defining a new functorial subgroup of a topological group $G$, namely, the intersection $o^{*}(G)$ of all open normal subgroups of a group $G$. If a locally compact group $L$ admits a base at the identity consisting of neighborhoods that are invariant under conjugation (that is, $L$ is so-called balanced or admits small invariant neighborhoods), which is the case for compact or abelian groups, then $o(L)=o^{*}(L)$. There are, however, many locally compact groups that do not have this property, a fact that explains our choice of terminology.

\section{Examples 2.8.}

(a) The semidirect product $L:=\{0,1\}^{\mathbb{Z}} \rtimes \mathbb{Z}$, where $\mathbb{Z}$ acts on the compact group $K:=\{0,1\}^{\mathbb{Z}}$ by shifts, is locally compact and zero-dimensional, and thus $o(L)$ is trivial. However, $K$ is the smallest open normal subgroup of $L$, and therefore $o^{*}(L)=K$.

(b) For $p \in \mathbb{P}$, let $\mathbb{Q}_{p}$ denote the (locally compact) field of $p$-adic numbers. The discrete multiplicative group $\mathbb{Q}^{\times}$of non-zero rationals acts on $\mathbb{Q}_{p}$ by multiplication. The semidirect product $L:=\left(\mathbb{Q}_{p},+\right) \rtimes \mathbb{Q}^{\times}$is locally compact 
and zero-dimensional (and so, again, $o(L)$ is trivial), but $\mathbb{Q}_{p}$ is the smallest open normal subgroup of $L$, and therefore $o^{*}(L)=\mathbb{Q}_{p}$.

(c) In general, let $G$ be a locally compact group and let $D$ be a subgroup of $\operatorname{Aut}(G)$ such that $G$ contains no proper $D$-invariant open subgroup, and put $L:=G \rtimes D$, where $D$ is equipped with the discrete topology. Then, by Theorem 1.1, the locally compact group $L$ has the property that $o(L)=L_{0}=G_{0}$ and $o^{*}(L)=G$.

\section{Proof of Theorem $\mathrm{A}$}

Theorem A. Let $G$ be a hereditarily locally pseudocompact group. Then $G / G_{0}$ is zero-dimensional and $G_{0}=q(G)=z(G)=o(G)$; that is, $G$ is strongly Vedenissoff.

In this section, we present the proof of Theorem $\mathrm{A}$. By Theorem 2.7(a),

$$
q(G)=z(G)=o(G)
$$

for every locally pseudocompact group $G$. We have already noted that if $G / G_{0}$ is zero-dimensional, then $G_{0}=q(G)=z(G)$. Thus, it suffices to show that $G / G_{0}$ is zero-dimensional for every hereditarily locally pseudocompact group $G$. Since every (Hausdorff) quotient of a hereditarily locally pseudocompact group is again hereditarily locally pseudocompact, and the quotient $G / G_{0}$ is hereditarily disconnected (cf. [19, 7.3] and [22, 1.32(c)]), it suffices to prove the following statement.

Theorem 3.1. Let $G$ be a hereditarily locally pseudocompact, hereditarily disconnected group. Then $\widetilde{G}$ is hereditarily disconnected, and $G$ is zero-dimensional.

In the setting of Theorem 3.1, if $\widetilde{G}$ is hereditarily disconnected, then by Theo$\operatorname{rem}$ 1.1, $\widetilde{G}$ is zero-dimensional (because it is locally compact), and so $G$ is zerodimensional too. Thus, it suffices to show that $\widetilde{G}$ is hereditarily disconnected whenever $G$ is so. We prove the contrapositive of this statement, namely, that if $(\widetilde{G})_{0}$ is non-trivial, then $G_{0}$ is non-trivial too. The proof is broken down into several steps: First, it is shown in Proposition 3.2 that Theorem 3.1 holds in the case where the completion $\widetilde{G}$ of $G$ is a direct product of a zero-dimensional compact group and the real line $\mathbb{R}$. Then, in Proposition 3.4, it is proven that if $\widetilde{G}$ contains a non-trivial compact connected subgroup, then $G_{0}$ is non-trivial. Finally, it is shown that if the component of $\widetilde{G}$ is non-trivial, but contains no compact connected subgroup, then $G_{0}$ contains $\mathbb{R}$ as a closed subgroup.

Proposition 3.2. Let $N$ be a zero-dimensional compact group, and $G$ a dense hereditarily locally pseudocompact subgroup of $N \times \mathbb{R}$. Then one has $\{e\} \times \mathbb{R} \subseteq G$.

In order to prove Proposition 3.2, we recall a notion and a result that is wellknown to profinite group theorists. A topological group $P$ is topologically finitely generated if it contains a dense finitely generated group, that is, if there exists a finite subset $F$ of $P$ such that $P=\operatorname{cl}_{P}\langle F\rangle$.

Theorem 3.3 ([29, 2.5.1], [15, 2.1(a)]). If $P$ is a topologically finitely generated compact zero-dimensional group, then $P$ is metrizable.

Proof of Proposition 3.2. As $G$ is locally pseudocompact, by Theorem 2.2, $G$ is $G_{\delta}$-dense in $N \times \mathbb{R}$. The set $A_{r}:=N \times\{r\}$ is a $G_{\delta^{-}}$set in $N \times \mathbb{R}$ for every $r \in \mathbb{R}$, and so $A_{r} \cap G \neq \emptyset$. Thus, for every $r \in \mathbb{R}$, there is $g \in N$ such that $(g, r) \in G$. 
Let $g_{1}, g_{2} \in N$ be such that $x_{1}:=\left(g_{1}, 1\right) \in G$ and $x_{2}:=\left(g_{2}, \sqrt{2}\right) \in G$, and put $P:=\operatorname{cl}_{N}\left\langle g_{1}, g_{2}\right\rangle$ and $H:=\operatorname{cl}_{G}\left\langle x_{1}, x_{2}\right\rangle$. Since $P$ is a closed subgroup of $N$, it is a compact topologically finitely generated zero-dimensional group, and by Theorem 3.3. $P$ is metrizable. Thus, the product $P \times \mathbb{R}$ is metrizable, and so $H$ is metrizable, being a subgroup of $P \times \mathbb{R}$. On the other hand, $H$ is locally pseudocompact, being a closed subgroup of the hereditarily locally pseudocompact group $G$. Therefore, $H$ is locally compact. Hence, by Theorem 2.1, $H$ is closed not only in $G$, but also in $N \times \mathbb{R}$.

Let $\pi_{2}: N \times \mathbb{R} \rightarrow \mathbb{R}$ denote the second projection, and put $\pi:=\pi_{2 \mid H}$. Since $N$ is compact and $H$ is closed in $N \times \mathbb{R}, \pi_{2}$ is a closed map (cf. [18, 3.1.16]), and thus $\pi(H)$ is closed in $\mathbb{R}$ and $\pi$ is a closed map too. This implies that $\pi$ is surjective, because $\pi(H)$ contains the dense subgroup $\langle 1, \sqrt{2}\rangle$ of $\mathbb{R}$. Consequently, $\pi$ is a quotient map, and $\mathbb{R}$ is topologically isomorphic to a quotient of $H$. Therefore, $H$ is not zero-dimensional, and by Theorem 1.1. $H_{0}$ is non-trivial.

Since $H_{0} \subseteq\{e\} \times \mathbb{R}=(N \times \mathbb{R})_{0}$, one has $H_{0}=\{e\} \times \mathbb{R}$, because $\mathbb{R}$ has no nontrivial proper connected subgroups. Hence, $\{e\} \times \mathbb{R}=H_{0} \subseteq H \subseteq G$, as desired.

Proposition 3.4. Let $G$ be a hereditarily locally pseudocompact group such that the completion $\widetilde{G}$ is $\sigma$-compact. If $\widetilde{G}$ contains a non-trivial compact connected subgroup, then $G_{0}$ is non-trivial.

Proof. Let $C$ be a non-trivial connected compact subgroup of $\widetilde{G}$. By Theorem2.3(c), $\Lambda_{c}^{*}(\widetilde{G})$ is a base at the identity for the $G_{\delta}$-topology on $\widetilde{G}$. So, we may pick $K \in \Lambda_{c}^{*}(\widetilde{G})$. Since $K$ is a normal subgroup of $\widetilde{G}$, the set $K C$ is a subgroup of $\widetilde{G}$, and $K C$ is compact, because both $K$ and $C$ are compact. Furthermore, $K C$ is $G_{\delta}$-open in $\widetilde{G}$, as it contains the $G_{\delta}$-set $K$. By Theorem 2.2, $G$ is $G_{\delta^{-}}$-dense in $\widetilde{G}$. By Theorem $2.3(\mathrm{a})$, the $G_{\delta}$-topology is a group topology on $\widetilde{G}$. Consequently, by Lemma 2.5, $P:=K C \cap G$ is $G_{\delta}$-dense in $K C$; in particular, $P$ is dense in $K C$, and thus, by Theorem 2.1, $\widetilde{P}=K C$.

We show that $P$ is hereditarily pseudocompact. Let $S$ be a closed subgroup of $P$. Since $K C$ is compact, it is closed in $\widetilde{G}$, and so $P$ is a closed subgroup of $G$. Thus, $S$ is a closed subgroup of $G$, and by our assumption, $S$ is locally pseudocompact. As $S$ is contained in the compact group $K C$, it is precompact. Consequently, by Theorem 2.2. $S$ is pseudocompact. This shows that $P$ is hereditarily pseudocompact. Therefore, by Theorem [1.3, $P_{0}$ is dense in $(\widetilde{P})_{0}=(K C)_{0}$, and hence

$$
\{e\} \neq C \subseteq(K C)_{0} \subseteq \mathrm{cl}_{K C} P_{0} \subseteq \mathrm{cl}_{\widetilde{G}} G_{0} .
$$

In particular, $G_{0}$ cannot be trivial, as desired.

One last ingredient of the proof of Theorem 3.1 is a result that is often referred to as Iwasawa's Theorem (although it also relies on the work of Yamabe).

Theorem 3.5 ([38, Theorem 5'], 21, Theorem 13]). Let $L$ be a connected locally compact group. Then there is a compact connected subgroup $C$ and closed subgroups $H_{1}, \ldots, H_{r}$ such that each $H_{i}$ is topologically isomorphic to the additive group $\mathbb{R}$, and $L$ is homeomorphic to $H_{1} \times \cdots \times H_{r} \times C$.

Proof of Theorem 3.1. We prove the contrapositive of the theorem. Let $G$ be a hereditarily locally pseudocompact group such that $(\widetilde{G})_{0} \neq\{e\}$. We show that $G_{0} \neq\{e\}$. 
Step 1 . As $G$ is locally precompact, its completion $L:=\widetilde{G}$ is locally compact. Let $U$ be a neighborhood of the identity in $L$ such that $\operatorname{cl}_{L} U$ is compact. Put $L^{\prime}:=\langle U\rangle$, the subgroup generated by $U$, and $G^{\prime}:=L^{\prime} \cap G$. We claim that by replacing $G$ with $G^{\prime}$ if necessary, we may assume that $\widetilde{G}$ is $\sigma$-compact from the outset.

Since $L^{\prime}$ contains $U$, it is an open subgroup of $L$, and thus it is also closed. Consequently, $G^{\prime}$ is open and closed in $G$, and $G^{\prime}$ is also hereditarily locally pseudocompact. By Lemma 2.5, one has $L^{\prime}=\mathrm{cl}_{L} G^{\prime}$, and so, by Theorem 2.1. $L^{\prime}=\widetilde{G^{\prime}}$. As $L^{\prime}$ is generated by $U$, it is compactly generated, and in particular, it is $\sigma$ compact (cf. [19, 5.12, 5.13]). Since $L^{\prime}$ is an open subgroup of $L$, by Theorem 1.1. $L_{0}=o(L) \subseteq L^{\prime}$, and therefore $L_{0}=L_{0}^{\prime}$ (because $L^{\prime} \subseteq L$ implies $\left.L_{0}^{\prime} \subseteq L_{0}\right)$. Similarly, one has $G_{0}=G_{0}^{\prime}$. Hence, it suffices to show that $G_{0}^{\prime} \neq\{e\}$, and by replacing $G$ with $G^{\prime}$ if necessary, we may assume that $\widetilde{G}$ is $\sigma$-compact.

Step 2. Since $\widetilde{G}$ is $\sigma$-compact, if $\widetilde{G}$ contains a non-trivial compact connected subgroup, then by Proposition 3.4 $G_{0}$ is non-trivial, and we are done. Thus, from now on, we assume that $\widetilde{G}$ contains no non-trivial compact connected subgroups.

If $N$ is a compact subgroup of $\widetilde{G}$, then $N_{0}=\{e\}$, and by Theorem 1.1, $N$ is zero-dimensional. Thus, every compact subgroup of $\widetilde{G}$ is zero-dimensional. In particular, $(\widetilde{G})_{0}$ contains no non-trivial compact connected subgroups. Therefore, by Theorem 3.5. our assumption $(\widetilde{G})_{0} \neq\{e\}$ yields that there is a closed subgroup $H$ of $\widetilde{G}$ such that $H \cong \mathbb{R}$ (that is, $H$ is topologically isomorphic to the additive group $\mathbb{R})$.

Step 3. By Theorem $2.3(\mathrm{c}), \Lambda_{c}^{*}(\widetilde{G})$ is a base at the identity for the $G_{\delta}$-topology on $\widetilde{G}$, and so we may pick $N \in \Lambda_{c}^{*}(\widetilde{G})$. Since $N$ is a normal subgroup, $(\widetilde{G})_{0}$ acts continuously on $N$ by conjugation, and the orbit of $x$ is a connected subspace of $N$. By Step 2, $N$ is zero-dimensional. Thus, the orbit of each $x \in N$ is a singleton. Therefore, $g^{-1} x g=x$ for every $g \in(\widetilde{G})_{0}$ and $x \in N$. In particular, the elements of $H$ and $N$ commute (elementwise). We note that this argument, concerning the commuting of connected and zero-dimensional normal subgroups, is due to K. H. Hofmann (cf. [20]).

Let $f: \mathbb{R} \rightarrow H$ be a topological isomorphism. The continuous surjection $h: N \times \mathbb{R} \rightarrow N H$ given by $h(x, r)=x f(r)$ is a homomorphism, because $f$ is a homomorphism, and $N$ and $H$ commute (elementwise). We show that $h$ is a topological isomorphism. Since $H \cong \mathbb{R}$, the only compact subgroup of $H$ is the trivial one, and thus $N \cap H=\{e\}$. Therefore, $h$ is injective. Since $N$ is compact and normal, and $H$ is closed in $\widetilde{G}$, the subgroup $N H$ is closed in $\widetilde{G}$, and so $N H$ is locally compact. The domain $N \times \mathbb{R}$ of $h$ is also locally compact and $\sigma$-compact. Consequently, by the Open Mapping Theorem, $h$ is open (cf. [19, 5.29]). Hence, $h$ is a topological isomorphism.

The subgroup $N H$ is $G_{\delta}$-open in $\widetilde{G}$, because it contains the $G_{\delta}$-set $N$. By Theorem 2.2 $G$ is $G_{\delta}$-dense in $\widetilde{G}$. By Theorem 2.3(a), the $G_{\delta}$-topology is a group topology on $\widetilde{G}$. So, by Lemma 2.5, $P:=N H \cap G$ is $G_{\delta}$-dense in $N H$; in particular, $P$ is dense in $N H$, and by Theorem 2.1. $\widetilde{P}=N H$. Since $P$ is a closed subgroup of $G$, it is hereditarily locally pseudocompact. Thus, $P^{\prime}:=h^{-1}(P)$ satisfies the conditions of Proposition 3.2. Consequently, $H=h(\{e\} \times \mathbb{R}) \subseteq h\left(P^{\prime}\right) \subseteq G$. Hence, $G_{0}$ is non-trivial, as desired. 


\section{Proof of Theorem D}

Theorem D, For every natural number $n$ or $n=\omega$, there exists an abelian pseudocompact group $G_{n}$ such that $G_{n}$ is perfectly totally minimal, hereditarily disconnected, but $\operatorname{dim} G_{n}=n$.

In this section, we prove Theorem $\mathrm{D}$ by establishing a general construction that allows one to "realize" minimal abelian groups as quasi-components of minimal pseudocompact groups. A weaker version of Theorem D, which provides totally minimal pseudocompact groups, was announced in [11, 1.4.2]. The novelty of Theorem $\mathrm{D}$, in addition to its complete proof, is that we obtain perfectly totally minimal pseudocompact groups.

Theorem $\mathbf{D}$. Let $A$ be a precompact abelian group that is contained in a connected compact abelian group $C$. Then there exists a pseudocompact abelian group $G$ such that $A \cong q(G)$ and $C \cong(\widetilde{G})_{0}$, and in particular, $\operatorname{dim} G=\operatorname{dim} C$. Furthermore, if $C=\widetilde{A}$ and

(a) $A$ is minimal, then $G$ may be chosen to be minimal;

(b) A is totally minimal, then $G$ may be chosen to be totally minimal;

(c) $A$ is perfectly minimal, then $G$ may be chosen to be perfectly minimal;

(d) A is perfectly totally minimal, then $G$ may be chosen to be perfectly totally minimal.

Theorem D follows a line of "embedding" results, which state that certain (locally) precompact groups embed into (locally) pseudocompact groups as a particular (e.g., functorial) closed subgroup (cf. [4, 2.1], [34, [5, 7.6], [35, 8, 3.6], and [3, 5.6]). The novelty is that minimality properties of the group $A$ are inherited by the group $G$ that is constructed. By the celebrated Prodanov-Stoyanov Theorem, every minimal abelian group is precompact (cf. [26] and [27]), and so the condition that the group $A$ is precompact is not restrictive at all.

We first show how Theorem $\mathrm{D}$ follows from Theorem $\mathrm{D}$ and then proceed to proving the latter. To that end, we recall a characterization due to Stoyanov for groups that are not only perfectly totally minimal, but their powers have the same property too (cf. 32]). For an abelian topological group $G$, let $w t d(G)$ denote the subgroup of elements $x$ in $G$ for which there exists a positive integer $m$ such that for every sequence $\left\{k_{n}\right\}_{n=1}^{\infty}$ of integers, one has $m^{n} k_{n} x \longrightarrow 0$ in $G$. In other words,

$$
w t d(G):=\left\{x \in G \mid \exists m>0, \forall\left\{k_{n}\right\}_{n=1}^{\infty} \in \mathbb{N}^{\omega}, m^{n} k_{n} x \longrightarrow 0\right\} .
$$

Theorem 4.1 (32], [14, 6.1.18]). Let $P$ be a precompact abelian group. Then $P^{\lambda}$ is perfectly totally minimal for every cardinal $\lambda$ if and only if $w t d(\widetilde{P}) \subseteq P$.

Proof of Theorem $\mathbb{D}$. Put $P:=\mathbb{Q} / \mathbb{Z}$. Then $\widetilde{P}=\mathbb{R} / \mathbb{Z}$ and $w t d(\widetilde{P})=\mathbb{Q} / \mathbb{Z}=P$, and by Theorem 4.1, $A_{n}:=P^{n}$ is perfectly totally minimal for every natural number $n$ or $n=\omega$, and $A$ is contained in the connected compact group $C_{n}:=(\mathbb{R} / \mathbb{Z})^{n}$. By Theorem $\mathrm{D}(\mathrm{d})$, there exists a perfectly totally minimal pseudocompact group $G_{n}$ such that $A_{n} \cong q\left(G_{n}\right)$ and $\operatorname{dim} G_{n}=\operatorname{dim} C_{n}=n$. Since

$$
\left(G_{n}\right)_{0} \subseteq q\left(G_{n}\right)_{0} \cong\left(A_{n}\right)_{0}=\{0\},
$$

the group $G_{n}$ is hereditarily disconnected, as desired.

We proceed now to proving Theorem $\mathrm{D}$. The proof has two ingredients: a zero-dimensional pseudocompact group $H$ with good minimality properties, and 
a discontinuous homomorphism $h: \widetilde{H} \rightarrow C$ with kernel $H$. The desired group $G$ will be the sum of the graph of $h$ and the group $A$ formed in the product $H \times C$.

Lemma 4.2. For every infinite cardinal $\lambda$, there exists a pseudocompact zerodimensional group $H$ such that:

(i) $H$ is perfectly totally minimal;

(ii) $r_{0}(\widetilde{H} / H) \geq 2^{\lambda}$.

Proof. Let $\mathbb{P}$ denote the set of prime integers, and for $p \in \mathbb{P}$, let $\mathbb{Z}_{p}$ denote the group of $p$-adic integers. Put $N:=\prod_{p \in \mathbb{P}} \mathbb{Z}_{p}^{\omega_{1}}$. We think of elements of $N$ as tuples $\left(x_{p, \alpha}\right)$, where $p \in \mathbb{P}$ and $\alpha<\omega_{1}$. We define three subgroups of $N$ :

(1) $E_{1}:=\bigoplus_{p \in \mathbb{P}} \mathbb{Z}_{p}^{\omega_{1}}$ consists of elements $x$ such that $(\exists \alpha)\left(x_{p, \alpha} \neq 0\right)$ only for finitely many primes $p$ (or equivalently, $E_{1}=w t d(N)$ );

(2) $E_{2}:=\prod_{p \in \mathbb{P}} S_{p}$, where $S_{p}$ is the $\Sigma$-product of $\omega_{1}$-many copies of $\mathbb{Z}_{p}$ (or equivalently, $E_{2}$ consists of elements $x$ such that all but countably many coordinates $x_{p, \alpha}$ of $x$ are zero);

(3) $E:=E_{1}+E_{2}$.

We claim that $H:=E^{\lambda}$ has the desired properties.

The group $E$ is $G_{\delta}$-dense in $N$, because it contains $E_{2}$, which is clearly $G_{\delta}$-dense. Thus, $H$ is $G_{\delta^{-}}$-dense in the compact group $N^{\lambda}$, and in particular, by Theorem 2.1, $\widetilde{H}=N^{\lambda}$. Therefore, by Theorem 2.2, $H$ is pseudocompact. The group $H$ is zerodimensional, being a subspace of the zero-dimensional group $N^{\lambda}$.

Since $E$ is $G_{\delta}$-dense in the compact group $N$, in particular, it is dense, and by Theorem [2.1 $N=\widetilde{E}$. Thus, $w t d(\widetilde{E})=w t d(N)=E_{1} \subseteq E$, and therefore by Theorem 4.1, $H=E^{\lambda}$ is perfectly totally minimal.

In order to prove that $r_{0}(\widetilde{H} / H) \geq 2^{\lambda}$, it suffices to show that $r_{0}(N / E) \geq 1$, as $\widetilde{H} / H \cong(N / E)^{\lambda}$. Letting $\Delta$ denote the "diagonal" subgroup of $N$, that is, the subgroup generated by $d$ such that $d_{p, \alpha}=1$ for every $p$ and $\alpha$, we prove that $E \cap \Delta=\{0\}$. In fact, we show a bit more, namely, that every element in $E$ has at least one zero coordinate. Let $x=y+z \in E$, where $y \in E_{1}$ and $z \in E_{2}$. By the definition of $E_{1}$, there exists $q \in \mathbb{P}$ such that $y_{q, \alpha}=0$ for every $\alpha<\omega_{1}$. Since $z \in E_{2}$, all but countably many coordinates of $z$ are zero. In particular, there exists $\gamma<\omega_{1}$ such that $z_{q, \gamma}=0$. Therefore, $x_{q, \gamma}=y_{q, \gamma}+z_{q, \gamma}=0$. Hence, $E \cap \Delta=\{0\}$ and $r_{0}(N / E) \geq 1$, as desired.

We consider the next lemma to be part of the folklore of pseudocompact abelian groups (cf. [2, 3.6, 3.10]), and we provide its proof only for the sake of completeness.

Lemma 4.3. Let $K_{1}$ and $K_{2}$ be compact topological groups, and let $h: K_{1} \rightarrow K_{2}$ be a surjective homomorphism such that $\operatorname{ker} h$ is $G_{\delta}$-dense in $K_{1}$. Then the graph $\Gamma_{h}$ of $h$ is a $G_{\delta}$-dense subgroup of the product $K_{1} \times K_{2}$, and in particular, $\Gamma_{h}$ is pseudocompact.

Proof. Let $B$ be a non-empty $G_{\delta}$-subset of $K_{1} \times K_{2}$. Without loss of generality, we may assume that $B$ is of the form $B_{1} \times B_{2}$, where $B_{i}$ is a $G_{\delta}$-set in $K_{i}$. Pick $x_{2} \in B_{2}$. Since $h$ is surjective, there is $x_{1} \in K_{1}$ such that $h\left(x_{1}\right)=x_{2}$. The translate $B_{1} x_{1}^{-1}$ is a non-empty $G_{\delta}$-set in $K_{1}$, and thus we may pick $x_{0} \in B_{1} x_{1}^{-1} \cap \operatorname{ker} h$, because ker $h$ is $G_{\delta}$-dense in $K_{1}$. Since $h\left(x_{0} x_{1}\right)=h\left(x_{1}\right)=x_{2}$, one obtains that $\left(x_{0} x_{1}, x_{2}\right) \in \Gamma_{h} \cap\left(B_{1} \times B_{2}\right)$. This shows that $\Gamma_{h}$ meets every $G_{\delta}$-set in $K_{1} \times K_{2}$. Therefore, by Theorem 2.1. $K_{1} \times K_{2}$ is the completion of $\Gamma_{h}$. Hence, by Theorem $2.2 . \Gamma_{h}$ is pseudocompact. 
A last, auxiliary, tool in the proof of Theorem $\mathrm{D}$ is the following observation.

Remark 4.4. Let $\mathcal{P}$ denote one of the following properties: minimal, totally minimal, perfectly minimal, perfectly totally minimal. If $G$ contains a dense subgroup with property $\mathcal{P}$, then $G$ also has property $\mathcal{P}$ (cf. [31, Theorem 2], 25], 11, Propositions 1 and 2], [13, [14, 2.5.1, 4.3.3], and [22, 3.21, 3.23]).

Proof of Theorem D. Put $\lambda=w(C)$, and let $H$ be the group provided by Lemma 4.2. Since $r_{0}(\widetilde{H} / H) \geq 2^{\lambda}$, the quotient $\widetilde{H} / H$ contains a free abelian group $F$ of rank $2^{\lambda}$. As $|C| \leq 2^{\lambda}$, one may pick a surjective homomorphism $h_{1}: F \rightarrow C$. The group $C$ is divisible, because it is compact and connected (cf. [19, 24.25]). Thus, $h_{1}$ can be extended to a surjective homomorphism $h_{2}: \widetilde{H} / H \rightarrow C$.

Let $h: \widetilde{H} \rightarrow C$ denote the composition of $h_{2}$ with the canonical projection $\widetilde{H} \rightarrow \widetilde{H} / H$. By Theorem 2.2, $H$ is $G_{\delta}$-dense in $\widetilde{H}$, because $H$ is pseudocompact. Thus, $\operatorname{ker} h$ is $G_{\delta}$-dense in $\widetilde{H}$, because $H \subseteq \operatorname{ker} h$. Clearly, $h$ is surjective. Therefore, by Lemma 4.3, the graph $\Gamma_{h}$ of $h$ is $G_{\delta}$-dense in the product $\widetilde{H} \times C$.

Put $G:=\Gamma_{h}+(\{0\} \times A)$. Since $\Gamma_{h}$ is $G_{\delta}$-dense in $H \times C$ and contained in $G$, the group $G$ is $G_{\delta}$-dense too. Thus, by Theorem 2.1, $\widetilde{G}=\widetilde{H} \times C$, and by Theorem 2.2. $G$ is pseudocompact. As $H$ is zero-dimensional, $(\widetilde{G})_{0}=\{0\} \times C$, and by Theorem 2.7(a), $q(G)=(\widetilde{G})_{0} \cap G=\{0\} \times A$.

We now check that $\operatorname{dim} G=\operatorname{dim} C$. Since $G$ is pseudocompact, by Theorem 2.2 , $\widetilde{G}=\beta G$, and so $\operatorname{dim} G=\operatorname{dim} \beta G=\operatorname{dim} \widetilde{G}$ (cf. [18, 7.1.17]). As $H$ is zero-dimensional and pseudocompact, by Theorem 2.7 (b), $\operatorname{dim} \widetilde{H}=0$. Thus, by Yamanoshita's Theorem, $\operatorname{dim} \widetilde{G}=\operatorname{dim} \widetilde{H}+\operatorname{dim} C=\operatorname{dim} C$ (cf. [39], 24, Corollary 2], and [14, 3.3.12]). Therefore, $\operatorname{dim} G=\operatorname{dim} C$.

We turn to the minimality properties of $G$. Suppose that $C=\widetilde{A}$. The group $G$ always contains the product $H \times A$, but in this case, $H \times A$ is dense in $\widetilde{G}=\widetilde{H} \times C$, and thus it is dense in $G$. Therefore, by Remark 4.4. $G$ inherits all minimality properties of $H \times A$. Since $H$ is perfectly totally minimal, the product $H \times A$ inherits all minimality properties of $A$. This shows (a)-(d).

One wonders whether the condition $C=\widetilde{A}$ is necessary for parts (a) $-(\mathrm{d})$ of Theorem D. If the resulting group $G$ is to be totally minimal, then the answer is positive. Dikranjan showed that if $G$ is a minimal pseudocompact abelian group, then $q(G)$ is dense in $(\widetilde{G})_{0}$ if and only if $G / q(G)$ is minimal (cf. [10, 1.7]), in which case $(\widetilde{G})_{0}$ is the completion of $q(G)$. This settles the question for (b) and (d). The following remark settles the question for (a) and (c).

Remark 4.5. We note (without a proof) that the techniques of Theorem $\mathrm{D}$ can also be used to construct, for every positive integer $n$ or $n=\omega$, a perfectly minimal pseudocompact $n$-dimensional group $G$ such that $G / q(G)$ is not minimal, and hence $q(G)$ is not dense in $(\widetilde{G})_{0}$.

\section{ACKNOWLEDGEMENTS}

This work has emerged from the joint work of one of the authors with W. W. Comfort on locally precompact groups; the authors wish to express their heartfelt gratitude to Wis Comfort for the helpful discussions and correspondence. The authors wish to thank Dragomir Djokovic for valuable correspondence. The authors are grateful to Karen Kipper for her kind help in proofreading this paper for 
grammar and punctuation. The authors wish to thank the anonymous referee for the wealth of constructive comments that led to an improved presentation of this paper.

\section{REFERENCES}

[1] B. Banaschewski. Minimal topological algebras. Math. Ann., 211:107-114, 1974. MR0357520 (50:9988)

[2] W. W. Comfort and J. Galindo. Extremal pseudocompact topological groups. J. Pure Appl. Algebra, 197(1-3):59-81, 2005. MR2123980 (2006a:22001)

[3] W. W. Comfort and G. Lukács. Locally precompact groups: (local) realcompactness and connectedness. J. Lie Theory, 20:347-374, 2010. MR2681374

[4] W. W. Comfort and J. van Mill. On the existence of free topological groups. Topology Appl., 29(3):245-265, 1988. MR953957 (90e:22001)

[5] W. W. Comfort and J. van Mill. Concerning connected, pseudocompact abelian groups. Topology Appl., 33(1):21-45, 1989. MR.1020981 (90k:54002)

[6] W. W. Comfort and K. A. Ross. Pseudocompactness and uniform continuity in topological groups. Pacific J. Math., 16:483-496, 1966. MR0207886 (34:7699)

[7] W. W. Comfort and F. J. Trigos-Arrieta. Locally pseudocompact topological groups. Topology Appl., 62(3):263-280, 1995. MR1326826 (96c:22002)

[8] D. Dikranjan. Connectedness and disconnectedness in pseudocompact groups. Rend. Accad. Naz. Sci. XL Mem. Mat. (5), 16:211-221, 1992. MR1205752 (94f:22001)

[9] D. Dikranjan. Dimension and connectedness in pseudo-compact groups. C. R. Acad. Sci. Paris Sér. I Math., 316(4):309-314, 1993. MR.1204295 (94c:22004)

[10] D. Dikranjan. Zero-dimensionality of some pseudocompact groups. Proc. Amer. Math. Soc., 120(4):1299-1308, 1994. MR1185278 (94f:54077)

[11] D. Dikranjan. Compactness and connectedness in topological groups. Topology Appl., 84(13):227-252, 1998. MR.1611234 (99b:54060)

[12] D. Dikranjan. Closure operators in topological groups related to von Neumann's kernel. Topology Appl., 153(11):1930-1955, 2006. MR2227037 (2006m:18005)

[13] D. Dikranjan and I. R. Prodanov. Totally minimal topological groups. Annuaire Univ. Sofia Fac. Math. Méc., 69:5-11 (1979), 1974/75. MR562518(81c:22003)

[14] D. Dikranjan, I. R. Prodanov, and L. N. Stoyanov. Topological groups, volume 130 of Monographs and Textbooks in Pure and Applied Mathematics. Marcel Dekker Inc., New York, 1990. Characters, dualities and minimal group topologies. MR.1015288 (91e:22001)

[15] D. Dikranjan, M. Tkačenko, and V. Tkachuk. Topological groups with thin generating sets. J. Pure Appl. Algebra, 145(2):123-148, 2000. MR.1733248(2000m:22003)

[16] D. Doïtchinov. Produits de groupes topologiques minimaux. Bull. Sci. Math. (2), 96:59-64, 1972. MR0308323(46:7437)

[17] E. K. van Douwen. The weight of a pseudocompact (homogeneous) space whose cardinality has countable cofinality. Proc. Amer. Math. Soc., 80(4):678-682, 1980. MR587954 (82a:54009)

[18] R. Engelking. General topology, volume 6 of Sigma Series in Pure Mathematics. Heldermann Verlag, Berlin, second edition, 1989. Translated from the Polish by the author. MR 1039321 (91c:54001)

[19] E. Hewitt and K. A. Ross. Abstract harmonic analysis. Academic Press Inc., New York, 1963. MR0156915 (28:158)

[20] K. H. Hofmann. Eine Bemerkung über die zentralen Untergruppen in zusammenhängenden topologischen Gruppen. Arch. Math., 9:33-38, 1958. MR0110762 (22:1630)

[21] K. Iwasawa. On some types of topological groups. Ann. of Math. (2), 50:507-558, 1949. MR0029911 (10:679a)

[22] G. Lukács. Compact-like topological groups, volume 31 of Research and Exposition in Mathematics. Heldermann Verlag, Berlin, 2009. MR2590018

[23] M. G. Megrelishvili. G-minimal topological groups. In Abelian groups, module theory, and topology (Padua, 1997), volume 201 of Lecture Notes in Pure and Appl. Math., pages 289299. Dekker, New York, 1998. MR,1651174 (99j:22002)

[24] P. S. Mostert. Sections in principal fibre spaces. Duke Math. J., 23:57-71, 1956. MR0075575 $(17: 771 \mathrm{f})$ 
[25] I. R. Prodanov. Precompact minimal group topologies and p-adic numbers. Annuaire Univ. Sofia Fac. Math., 66:249-266 (1974), 1971/72. MR0412323 (54:449)

[26] I. R. Prodanov and L. N. Stojanov. Every minimal abelian group is precompact. C. R. Acad. Bulgare Sci., 37(1):23-26, 1984. MR748738 (85k:22007)

[27] I. R. Prodanov and L. N. Stojanov. Minimal group topologies. In Topology, theory and applications (Eger, 1983), volume 41 of Colloq. Math. Soc. János Bolyai, pages 493-508. NorthHolland, Amsterdam, 1985. MR863933 (87k:22001)

[28] D. Raǐkov. On the completion of topological groups. Bull. Acad. Sci. URSS. Sér. Math. [Izvestia Akad. Nauk SSSR], 10:513-528, 1946. MR.0020083 (8:500g)

[29] L. Ribes and P. Zalesskii. Profinite groups, volume 40 of Ergebnisse der Mathematik und ihrer Grenzgebiete. 3. Folge. A Series of Modern Surveys in Mathematics [Results in Mathematics and Related Areas. 3rd Series. A Series of Modern Surveys in Mathematics]. Springer-Verlag, Berlin, 2000. MR:1775104 (2001k:20060)

[30] W. Roelcke and S. Dierolf. Uniform structures on topological groups and their quotients. McGraw-Hill International Book Co., New York, 1981. Advanced Book Program. MR 644485 (83j:22001)

[31] R. M. Stephenson, Jr. Minimal topological groups. Math. Ann., 192:193-195, 1971. MR0286934 (44:4141)

[32] L. N. Stojanov. On products of minimal and totally minimal groups. In Proc. 11th Conf. of the Union of Bulg. Math., Sunny Beach, 1982, pages 287-294, 1982.

[33] M. G. Tkačenko. On dimension of locally pseudocompact groups and their quotients. Comment. Math. Univ. Carolin., 31(1):159-166, 1990. MR1056183(91e:54091)

[34] M. I. Ursul. Embedding of locally precompact groups into locally pseudocompact groups. Izv. Akad. Nauk Moldav. SSR Ser. Fiz.-Tekhn. Mat. Nauk, (3):54-56, 77, 1989. MR1049014 (91f:22005)

[35] M. I. Ursul. Embedding of precompact groups into pseudocompact groups. Izv. Akad. Nauk Moldav. SSR Mat., (1):88-89, 93, 1991. MR1130025 (92g:22003)

[36] N. Vedenissoff. Remarques sur la dimension des espaces topologiques. Uchenye Zapiski Moskov. Gos. Univ. Matematika, 30:131-140, 1939. MR0002526 (2:69i)

[37] A. Weil. Sur les Espaces à Structure Uniforme et sur la Topologie Générale. Publ. Math. Univ. Strasbourg. Hermann, Paris, 1937.

[38] H. Yamabe. A generalization of a theorem of Gleason. Ann. of Math. (2), 58:351-365, 1953. MR0058607 (15:398d)

[39] T. Yamanoshita. On the dimension of homogeneous spaces. J. Math. Soc. Japan, 6:151-159, 1954. MR0065566 (16:447a)

Department of Mathematics and Computer Science, University of Udine, Via delle Scienze, 208 - Loc. Rizzi, 33100 Udine, Italy

E-mail address: dikranja@dimi.uniud.it

Department of Mathematics, University of Manitoba, Winnipeg, Manitoba, R3T 2N2, CANADA

E-mail address: lukacs@cc.umanitoba.ca 